https://doi.org/10.22319/rmcp.v9i3.4295

Nota de investigación

\title{
Retención de membranas fetales y patologías uterinas en vacas lecheras tratadas con PGF2 $\alpha$ después del parto
}

\section{Retention of fetal membranes and uterine pathologies in dairy cows treated with PGF2 $\alpha$ after parturition}

Luis Ángel Valdés Pérez ${ }^{\mathrm{a}}$

Joel Hernández Cerón ${ }^{\mathrm{a}^{*}}$

Rodolfo Luzbel de la Sota ${ }^{\mathrm{b}}$

Carlos Fernando Aréchiga Flores ${ }^{\mathrm{c}}$

Eligio Gabriel Salgado Hernández ${ }^{\mathrm{a}}$

Antonio Romero Arredondo ${ }^{\mathrm{d}}$

${ }^{a}$ Facultad de Medicina Veterinaria y Zootecnia. Universidad Nacional Autónoma de México. México.

${ }^{\mathrm{b}}$ Facultad de Ciencias Veterinarias. Universidad Nacional de la Plata. La Plata, Argentina.

${ }^{\mathrm{c}}$ Facultad de Medicina Veterinaria y Zootecnia. Universidad Autónoma de Zacatecas. México.

${ }^{\mathrm{d}}$ Práctica profesional privada. México.

Autor de correspondencia: jhc@unam.mx

\section{- Resumen:}

Se probó si la administración de dos inyecciones de PGF2 $\alpha$ en las primeras 48 h posparto disminuye la incidencia de retención de las membranas fetales (RMF) y de patologías uterinas en vacas lecheras. Se utilizaron 800 vacas de diferente número de partos, las cuales se asignaron aleatoriamente a dos tratamientos; PGF2 $\alpha(n=400)$ recibieron una inyección de 
PGF $2 \alpha(500 \mu \mathrm{g}$ de cloprostenol sódico) en las primeras $12 \mathrm{~h}$ posparto y una segunda inyección $48 \mathrm{~h}$ después; testigo $(\mathrm{n}=400)$ no recibió PGF2 $\alpha$. Se registró la incidencia de RMF (vacas que no eliminaron la placenta de las siguientes $24 \mathrm{~h}$ posparto) y de patologías uterinas (metritis y endometritis), tasa de gestación en el primer servicio y porcentaje de vacas gestantes en el día 150 posparto. Las vacas testigo tuvieron mayor probabilidad de RMF (Odds ratio 1.69; $P=0.01$ ) que las vacas tratadas con PGF2 $\alpha$. La proporción de vacas con patologías uterinas en el día 28 posparto fue menor $(P=0.01)$ en las vacas tratadas con PGF2 $\alpha$ $(29.8 \%)$ que en las testigo (36.7 \%). La tasa de gestación en el día 150 posparto fue mayor en las vacas con involución normal que en las vacas con patologías uterinas (Odds ratio 1.4, $P=0.03$ ). Se concluye que dos inyecciones de PGF $2 \alpha$ en las primeras $48 \mathrm{~h}$ posparto (primeras 12 y $48 \mathrm{~h}$ posparto) disminuyen la incidencia de RMF y de patologías uterinas en vacas lecheras.

- Palabras clave: Vacas lecheras, Retención de membranas fetales, PGF2 $\alpha$.

\section{- Abstract:}

In order to determine its effect on incidence of retained fetal membranes (RFM) and postpartum uterine pathologies in dairy cows, the administration of two injections of PGF2 $\alpha$ in the first $48 \mathrm{~h}$ after parturition was tested. In this study, 800 cows with different parities were randomly assigned to two treatment groups: PGF $2 \alpha(n=400)$, receiving two postpartum PGF2 $\alpha$ injections ( $500 \mu \mathrm{g}$ of cloprostenol sodium), one during the first $12 \mathrm{~h}$ and one at $48 \mathrm{~h}$; and control $(n=400)$, not receiving PGF2 $\alpha$. The incidence of RFM (cows that had not expelled the placenta at $24 \mathrm{~h}$ postpartum), and of postpartum uterine pathologies (metritis and endometritis), was registered, as well as pregnancy rate at first service and percentage of pregnant cows at $\mathrm{d} 150$ postpartum. Control cows had a higher probability of presenting RFM (Odds ratio 1.69; $P=0.01$ ) than cows treated with PGF2 $\alpha$. Likewise, the proportion of cows with uterine pathologies at $\mathrm{d} 28$ postpartum was lower $(P=0.01)$ in cows treated with PGF2 $\alpha$ $(29.8 \%)$ than in control cows ( $36.7 \%)$. The accumulated pregnancy rate at $\mathrm{d} 150$ postpartum was higher in cows with normal involution than in cows with uterine pathologies (Odds ratio $1.4, P=0.03$ ). It is concluded that two injections of PGF2 $\alpha$ in the first $48 \mathrm{~h}$ postpartum (at 12 and $48 \mathrm{~h}$ ) lowers the incidence of RFM and of uterine pathologies in dairy cows.

- Key words: Dairy cows, Retention of fetal membranes, PGF2 $\alpha$.

Recibido: 11/10/2016

Aceptado: 07/11/2017 
La recuperación de la competencia reproductiva posparto en las vacas lecheras depende principalmente del tiempo que tome la involución uterina y del inicio de la actividad ovárica $^{(1)}$. Las patologías uterinas posparto incrementan el periodo del parto al primer servicio, generan bajas tasas de gestación en el primer servicio, incrementan el número de días abiertos, aumentan el número de vacas eliminadas y provocan importantes pérdidas económicas por baja en la producción y por el costo de los tratamientos ${ }^{(1,2)}$. Entre los principales factores de riesgo de las patologías uterinas posparto, está la retención de las membranas fetales $(\mathrm{RMF})^{(1,3,4)}$.

Dentro de las estrategias para prevenir y tratar las patologías uterinas se ha considerado el uso de la prostaglandina F2 $\alpha$ (PGF2 $\alpha$ ). La evaluación del uso de la PGF2 $\alpha$ como herramienta de manejo en la prevención de patologías uterinas ha arrojado resultados contradictorios ${ }^{(5)}$. Uno de los primeros hallazgos que demuestran la importancia de las PGF2 $\alpha$ en la involución uterina, es la correlación negativa entre las concentraciones de PGF2 $\alpha$ posparto con el tiempo de involución uterina ${ }^{(6,7)}$. Gross et $a l^{(8)}$ encontraron un efecto favorable de la administración de PGF2 $\alpha$ inmediatamente después del parto en la disminución de la incidencia de RMF en animales a los cuales se les indujo el parto con dexametasona; sin embargo, estos resultados contrastan con lo obtenido en otro estudio similar ${ }^{(9)}$.

Ortega et $a l^{(10)}$, observaron que la administración de dos inyecciones de prostaglandinas en las primeras $48 \mathrm{~h}$ posparto, disminuyó el porcentaje de vacas que presentaron RMF, redujo el tiempo del parto al primer estro, y aumentó el número de vacas gestantes en el día 90 posparto; sin embargo, no hay otro estudio que corrobore dichos resultados. Por tanto, en el presente trabajo se probó si la administración de dos inyecciones de PGF2 $\alpha$ en las primeras $48 \mathrm{~h}$ posparto (primeras 12 y $48 \mathrm{~h}$ posparto), disminuye la incidencia de RMF y de patologías uterinas en vacas lecheras.

El presente trabajo se realizó durante los meses de agosto a noviembre de 2014, en un establo lechero de manejo intensivo de 3,200 vacas en ordeño, con una producción promedio de 10,000 kg por lactación. Se utilizaron 800 vacas de diferente número de partos alimentadas con una ración integral, según las recomendaciones del $\mathrm{NRC}^{(11)}$.

Veintiún días antes del parto las vacas se trasladaron a un corral de acuerdo con su número de parto (primíparas o multíparas). Después del parto, las vacas se alojaron en el corral de vacas frescas de acuerdo, también, con su número de parto, en los cuales permanecieron hasta el día 21 posparto (pp).

Todas las vacas recibieron sistemáticamente tres inyecciones de PGF2 $\alpha$ con un intervalo de 14 días a partir del día $26 \pm 3 \mathrm{pp}$. Las vacas que se detectaron en estro después del periodo voluntario de espera (45 días), se inseminaron. Los animales no detectados en estro se enrolaron en un protocolo de sincronización e inseminación artificial a tiempo fijo 
(CoSynch). El diagnóstico de gestación se realizó por medio de palpación transrectal entre los días 40 y 50 post inseminación.

El manejo de los animales durante el experimento fue aprobado por el Subcomité Institucional para el Cuidado y Uso de Animales Experimentales (SICUAE) del Posgrado en Ciencias de la Producción y de la Salud Animal de la Universidad Nacional Autónoma de México.

Diez días antes del parto las vacas se asignaron aleatoriamente a dos diferentes tratamientos: PGF2 $\alpha(\mathrm{n}=400)$, recibieron $500 \mu \mathrm{g}$ de Cloprostenol sódico (Celosil ${ }^{\circledR}$; MSD Animal Health, México) vía intramuscular dentro de las primeras $12 \mathrm{~h}$ posparto ( 1 a $12 \mathrm{~h}$ ) y una segunda inyección $48 \mathrm{~h}$ después; testigo $(\mathrm{n}=400)$, no recibieron PGF2 $\alpha$. La proporción de vacas primíparas $(33 \%)$ y multíparas $(67 \%)$, la condición corporal al parto $(3.8 \pm 0.3)$, la proporción de partos gemelares $(2.9 \%)$ y partos asistidos $(44 \%)$ fueron similares entre las vacas tratadas con PGF2 $\alpha$ y las testigo $(P>0.10)$.

Todas las vacas se examinaron por vía transrectal en los días 14, 21 y 28 posparto, para determinar el grado de involución y hacer el diagnóstico de patologías uterinas. Se registró la incidencia de patologías uterinas de acuerdo con el criterio de Sheldon et al $l^{(12)}$. Se consideró como RMF a aquellas vacas que no eliminaron la placenta en las siguientes $24 \mathrm{~h}$ posparto; metritis, vacas con retraso en la involución uterina con descargas purulentas (primeros 21 días pp), sin signos de enfermedad sistémica, y endometritis vacas con descargas mucopurulentas ( 50 pus $\%$ y $50 \%$ moco) (después de 21 días pp). A los animales que resultaron positivos a cualquiera de las patologías descritas, se les aplicaron los tratamientos farmacológicos utilizados en el hato. Se evaluó el tiempo trascurrido del parto al primer servicio; tasa de gestación en el primer servicio (proporción de vacas gestantes del total inseminado) y tasa de gestación acumulada en el día 150 posparto.

El efecto de cada factor en la presentación de RMF se determinó por medio de regresión logística múltiple. La RMF se consideró como variable dependiente, mientras que el tratamiento, tipo de parto (simple o gemelar), dificultad de parto (asistido o no asistido), número de partos (primíparas o multíparas), sexo de la cría (macho o hembra) se consideraron como variables independientes ${ }^{(13)}$. La tasa de gestación en el primer servicio y la tasa de gestación acumulada en el día 150 posparto, también se analizaron mediante modelos de regresión logística múltiple considerando, además de la variables independientes mencionadas anteriormente, a la RMF (vacas con o sin RMF) y la salud uterina al día 28 posparto [involución normal o patologías uterinas (metritis o endometritis)]. El modelo se estableció de acuerdo al método propuesto por Hosmer y Lemeshow ${ }^{(14)}$ de modo análogo al usado por López-Gatius et al ${ }^{(15)}$. El modelo final se construyó mediante la técnica de eliminación manual paso a paso, que comprende: la selección preliminar de todas la variables para las asociaciones univariadas; la construcción de un modelo completo utilizando las variables significativas que resultaron del análisis univariado; la eliminación gradual de las 
variables no significativas del modelo completo; la comparación del modelo reducido con el modelo anterior para el ajuste del modelo y los factores de confusión; la evaluación de las interacciones entre las variables y la evaluación del ajuste del modelo mediante estadística de Hosmer-Lemeshow ${ }^{(14)}$. En el análisis univariado el valor de $P<0.25$ se utilizó como criterio de inclusión en el modelo inicial y se continuó depurando el modelo dejando sólo los efectos principales $(P<0.05)$.

La proporción de vacas con metritis o endometritis en los días 14, 21 y 28 posparto de los grupos tratado con PGF2 $\alpha$ y testigo, así como entre vacas con RMF y sin RMF, se comparó mediante un análisis de sobrevivencia (Kaplan-Meier). Las curvas se compararon mediante la prueba de Log-rank. Los días al primer servicio, se analizaron mediante un análisis de varianza (ANOVA).

El tratamiento con PGF2 $\alpha$ en las primeras horas posparto redujo la incidencia de RMF (17.5 vs $11 \%$; testigo y PGF2 $\alpha$, respectivamente; razón de probabilidades 1.69; intervalo de confianza $95 \%=1.12-2.55 ; P<0.01)$. La disminución de la incidencia de RMF observada en este trabajo concuerda con lo encontrado por Ortega et $a l^{(10)}$, quienes aplicaron el mismo tratamiento en condiciones similares a las del presente estudio y obtuvieron una reducción en la RMF (3 vs $10 \%$; vacas tratadas y testigo, respectivamente). Cabe destacar que en el presente estudio la reducción de la RMF fue de $37 \%$ con respecto a las vacas testigo, mientras que en el trabajo de Ortega et $a l^{(10)}$ fue de $70 \%$. No obstante, los resultados son alentadores, ya que muestran que aún en un hato con incidencia elevada de RMF (17.5\%), en el cual habría más factores de riesgo de $\mathrm{RMF}^{(16)}$, el tratamiento con PGF2 $\alpha$ reduce esta patología.

El mecanismo por el cual la administración de PGF2 $\alpha$ en las primeras horas posparto reduce la RMF se desconoce. En algunos estudios se ha propuesto que la PGF2 $\alpha$ facilita el proceso de separación y expulsión de la placenta; así, se ha encontrado a nivel caruncular una alteración en la síntesis de PGF2 $\alpha$ en vacas con $\mathrm{RMF}^{(17)}$. Además, la relación de PGF2 $\alpha$ :PGE2 en las vacas que no presentan RMF es mayor a favor de la PGF2 $\alpha$, lo que indica la importancia de altos niveles de esta hormona a nivel caruncular para la expulsión de la placenta ${ }^{(18,19)}$. Dentro de la patogenia de la RMF, se destaca el papel de los neutrófilos; se menciona que existe una alteración en la actividad de los neutrófilos a nivel uterino en aquellos animales que presentan $\mathrm{RMF}^{(20,21)}$. Se ha observado que la inducción del parto con dexametasona es un factor de riesgo para la RMF, ya que este tratamiento inhibe la actividad de los neutrófilos en la unión de la carúncula y el cotiledón ${ }^{(16)}$. Cabe señalar que en el presente estudio, la inyección que pudo influir en la eliminación de la placenta fue la que se aplicó en las primeras $12 \mathrm{~h}$ posparto, ya que al momento de la segunda inyección ( $48 \mathrm{~h}$ después) ya se había determinado qué vacas tuvieron RMF; es prudente mencionar que el tiempo de corte para diagnosticar un caso de RMF fue a las $24 \mathrm{~h}$ posparto. No obstante, el tratamiento con las dos inyecciones de PGF2 $\alpha$ tuvo efectos en otras variables reproductivas que se discuten más adelante. 
Un mecanismo del efecto favorable de la administración de PGF2 $\alpha$ en la RMF propuesto por Salgado et $a l^{(22)}$, está asociado con las concentraciones sanguíneas de calcio. Así, en su estudio las vacas que recibieron PGF $2 \alpha$ en un tratamiento similar al del presente trabajo, tuvieron mayores concentraciones circulantes de calcio en los primeros siete días posparto que las vacas testigo. Estos investigadores proponen que la administración de PGF2 $\alpha$ incrementa la calcemia, lo cual subsana parcialmente las hipocalcemia subclínica que padecen las vacas lecheras durante los primeros días posparto ${ }^{(23)}$. Estos hallazgos tendrían relación con el proceso de expulsión de la placenta y con las patologías uterinas, ya que se ha encontrado mayor incidencia de patologías uterinas en vacas con hipocalcemia ${ }^{(24,25)}$. El efecto negativo de los bajos niveles de calcio posparto, puede explicarse por la disminución de la actividad fagocitaria de los neutrófilos en vacas con hipocalcemia ${ }^{(25)}$; asimismo, los neutrófilos liberan proteasas y colagenasa que favorecen la expulsión de la placenta, procesos calcio dependientes ${ }^{(26)}$. No obstante el desconocimiento del mecanismos de acción de la inyección de PGF2 $\alpha$, la obtención de una respuesta positiva en la incidencia de RMF similar a la observada por Ortega et $a l^{(10)}$ ofrece bases consistentes para realizar futuras investigaciones que expliquen en qué procesos influye la PGF2 $\alpha$ para facilitar la expulsión de la placenta.

Por otro lado, se observó en el presente estudio que el parto gemelar incrementa la probabilidad de presentar RMF (razón de probabilidades 5.19; intervalo de confianza 95\%= 2.5-13.85; $P<0.01$ ), lo cual es congruente con la información publicada, ya que la manipulación uterina durante el parto gemelar incrementa el riesgo de $\mathrm{RMF}^{(27)}$. Sin embargo, la asistencia en el parto no tuvo un efecto significativo en la $\operatorname{RMF}(P>0.05)$, lo cual es diferente a lo esperado, ya que la distocia también es un factor de riesgo de $\mathrm{RMF}^{(16)}$. Cabe mencionar que el dato de distocias no aparece en los registros del hato, sólo se anota asistencia en el parto independientemente del grado de ayuda; así, la asistencia al parto registrada fue de $43.7 \%$ en el grupo tratado con PGF $2 \alpha$ y $45.2 \%$ en el testigo.

De acuerdo con el análisis de Kaplan-Meyer, las vacas tratadas con PGF2 $\alpha$ tuvieron menor prevalencia de metritis o endometritis (Figura 1; $P<0.05$ ) que las vacas testigo (90.3 vs 91.5; 70.5 vs 73.5; 29.8 vs 36.7; en los días 14, 21 y 28, respectivamente), lo cual pudo ser un efecto de la menor incidencia de RMF observada en las vacas tratadas con PGF2 $\alpha$. Esta posibilidad es congruente con los resultados del análisis de sobrevivencia realizado en vacas con RMF y sin RMF, en el cual se observa que las vacas con RMF tuvieron mayor prevalencia de patologías uterinas que las vacas sin RMF (Figura 2; $P<0.01$ ); se menciona que la RMF es uno de los principales factores de riesgo de metritis y endometritis ${ }^{(3,4)}$. 
Figura 1: Curva de sobrevivencia para la proporción de vacas con patologías uterinas en los primeros 28 días posparto tratadas con prostaglandinas (PGF2 $\alpha$ )

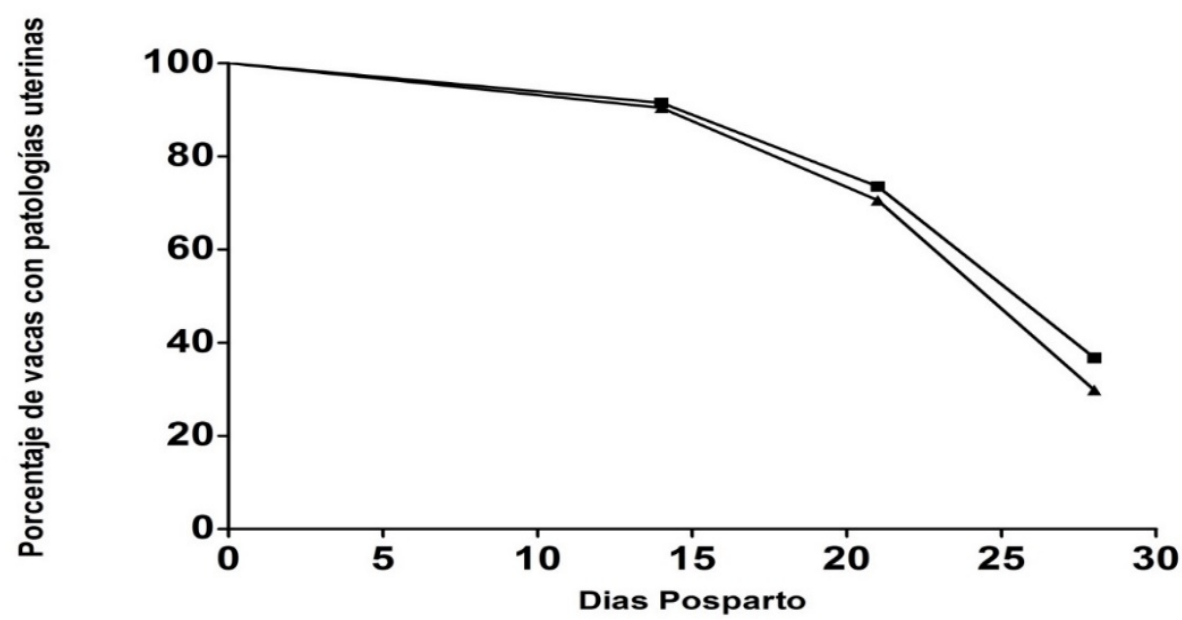

Una inyección de PGF2a en las primeras $12 \mathrm{~h}$ posparto y una segunda inyección

$$
\text { a las } 48 \mathrm{~h} \text { posparto }(-\mathbf{\Lambda}-) \text {, y testigos ( } \rightarrow-) ; P<0.05 \text {. }
$$

Figura 2: Curva de sobrevivencia para la proporción de vacas con patologías uterinas en los primeros 28 días posparto en vacas con RMF ( $-\boldsymbol{-}-)$ y $\sin \mathrm{RMF}(\boldsymbol{- \Delta}-) ; P<0.01$

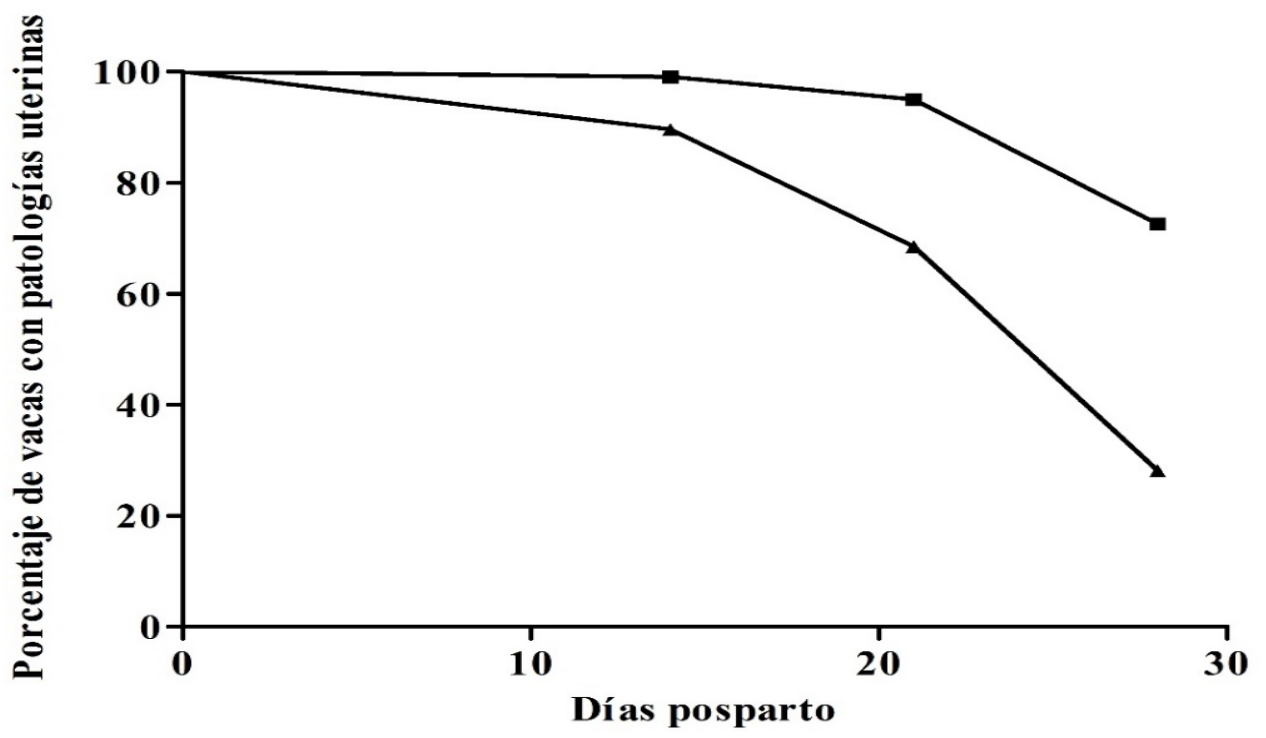

El tratamiento con PGF2 $\alpha$ no afectó la probabilidad de gestación en el primer servicio. Las vacas de parto asistido tuvieron la misma probabilidad de quedar gestantes en su primer servicio en comparación con las vacas de parto no asistido (Cuadro1). Las variables sexo de la cría, tipo de puerperio, RMF, tipo de parto y su interacción con el tratamiento no afectaron la probabilidad de gestación en el primer servicio $(P>0.10)$. 
Cuadro 1: Razón de probabilidades (RP) para la gestación en el primer servicio de acuerdo a las variables en el modelo de regresión logística

\begin{tabular}{llccccc}
\hline Variables & Clases & $\mathbf{n}$ & Tasa de gestación & RP & IC 95\% & $\boldsymbol{P}$ \\
\hline Tratamiento & Testigo & 344 & 24.1 & Referencia & & \\
& PGF2a & 350 & 22.8 & 0.91 & $0.64-1.30$ & 0.62 \\
Dificultad al & No asistido & 389 & 24.9 & 1.38 & $0.95-2.00$ & 0.08 \\
Parto & Asistido & 305 & 21.6 & Referencia & & \\
Número de & Primíparas & 236 & 31.3 & 2.02 & $1.40-2.92$ & 0.01 \\
partos & Multíparas & 458 & 19.4 & Referencia & & \\
\hline
\end{tabular}

El tratamiento con PGF2 $\alpha$ no afectó la tasa acumulada de gestación en el día 150 posparto. Las vacas que padecieron patologías uterinas en el día 28 posparto tuvieron menor probabilidad de estar gestantes en el día 150 posparto (Cuadro 2). El mecanismo por el cual una infección uterina posparto afecta negativamente el establecimiento de la gestación no está determinado; algunas evidencias muestran efectos nocivos de las endotoxinas en el endometrio, en el desarrollo folicular y en el desarrollo embrionario ${ }^{(28)}$. Además, las vacas con patologías uterinas tienen mayor riesgo de padecer endometritis subclínica, la cual afecta negativamente la tasa de gestación en el primer servicio e incrementa el riesgo de pérdidas embrionarias y fetales ${ }^{(29)}$. Los factores como sexo de la cría, dificultad de parto y tipo de parto no afectaron la probabilidad de gestación acumulada en el día 150 posparto, ni hubo interacción con el tratamiento $(P>0.10)$.

Cuadro 2: Razón de probabilidades (RP) para la gestación en el día 150 posparto de acuerdo a las variables en el modelo de regresión logística

\begin{tabular}{lcccccc}
\hline Variables & Clases & $\mathbf{n}$ & Tasa de gestación & RP & IC 95\% & $\boldsymbol{P}$ \\
\hline Tratamiento & Testigo & 336 & 59.8 & Referencia & & \\
& PGF2a & 341 & 62.4 & 1.10 & $0.80-1.50$ & 0.53 \\
Número de & Primíparas & 236 & 67.3 & 1.47 & $1.06-2.06$ & 0.02 \\
parto & Multíparas & 441 & 57.8 & Referencia & & \\
Salud uterina* & Normal & 377 & 64.9 & 1.40 & $1.02-1.92$ & 0.03 \\
& Patologías & 300 & 56.3 & Referencia & & \\
Tipo de parto & Simple & 657 & 61.9 & 2.46 & $0.96-6.34$ & 0.06 \\
& Gemelar & 20 & 35.0 & Referencia & & \\
\hline
\end{tabular}

* Día 28 posparto.

El intervalo del parto al primer servicio fue similar $(P>0.05)$ entre las vacas tratadas con PGF $2 \alpha$ y las testigo $[68 \pm 1.1$ vs $65 \pm 1.1$ días (media \pm error estándar); grupos PGF2 $\alpha$ y testigo, respectivamente]. Se esperaba, sin embargo, que los efectos en la salud uterina 
provocados por las dos inyecciones de PGF2 $\alpha$ se reflejaran en menor intervalo del parto al primer servicio. No obstante, cuando se compararon los grupos con involución normal o patológica en el día 28 posparto independientemente de si fueron tratados o no con PGF2 $\alpha$, las vacas con patologías uterinas tuvieron mayor intervalo al primer servicio ( $71 \pm 1.1$ días; $P<0.01)$ en comparación con las vacas con involución normal (62 \pm 1.0 días), lo cual es congruente con los efectos que tienen las infecciones uterinas en el inicio de la actividad ovárica posparto y en la salud uterina al momento del primer servicio ${ }^{(1,5)}$.

Las vacas de primer parto fueron más fértiles que las vacas multíparas (Cuadros 1 y 2); así, tanto la tasa de gestación en el primer servicio, como la tasa acumulada de gestación en el día 150 posparto fue mayor en las vacas primíparas que en las multíparas. Esta observación es congruente con otros estudios ${ }^{(30,31)}$.

Se concluye que dos inyecciones de PGF2 $\alpha$, en las primeras $48 \mathrm{~h}$ posparto (primeras 12 y 48 horas posparto) disminuyen la incidencia de retención de membranas fetales y de patologías uterinas en vacas lecheras.

\section{Agradecimientos}

Este estudio fue financiado en parte por el proyecto IN219811-3 y por el PASPA-DGAPA de la Universidad Nacional Autónoma de México. Se agradece al propietario del Rancho Montoro (Aguascalientes, México), al personal Médico y a los trabajadores, su apoyo para realizar este estudio.

\section{- Literatura citada:}

1. Galvâo KN. Postpartum uterine diseases in dairy cows. Anim Reprod 2012;9:290-296.

2. Sheldon IM, Cronin J, Goetze L, Donofrio G, Schuberth HJ. Defining postpartum uterine disease and the mechanisms of infection and immunity in the female reproductive tract in cattle. Biol Reprod 2009;81:1025-1032. 
3. Dubuc J, Duffield TF, Leslie KE, Walton JS, LeBlanc SJ. Risk factors for postpartum uterine diseases in dairy cows. J Dairy Sci 2010;93:5764-5771.

4. Potter TJ, Guitian J, Fishwick J, Gordon PJ, Sheldon IM. Risk factors for clinical endometritis in postpartum dairy cattle. Theriogenology 2010;74:127-134.

5. LeBlanc SJ. Postpartum uterine diseases and dairy herd reproductive performance: A review. Vet J 2008;176:102-114.

6. Lindell JO, Kindahl H, Jansson L, Edqvist LE. Post-partum release of prostaglandin F2 $\alpha$ and uterine involution in the cows. Theriogenology 1982;17:237-245.

7. Madej A, Kindahl H, Woyno W, Edqvist LE, Stupnicki R. Blood levels of 15-keto13,14-dihydro-prostaglandin F2 $\alpha$ during the postpartum period in primiparous cows. Theriogenology 1984;21:279-287.

8. Gross TS, Williams WF, Moreland TW. Prevention of the retained fetal membrane syndrome (retained placenta) during induced calving in dairy cattle. Theriogenology 1986;26:365-370.

9. Garcia A, Barth AD, Mapletoft RJ. The effects of treatment with cloprostenol or dinoprost within one hour of induced parturition on the incidence of retained placenta in cattle. Can Vet J 1992;33:175-183.

10. Ortega A, López R, Mapes G, Ortiz O, Hernández CJ. Uterine pathologies and fertility of dairy cows treated with two injections of PGF $2 \alpha$ within the first 48 hours after calving. Vet Méx 2012;43:235-240.

11. National Research Council. 2001. Nutrient requirements of dairy cattle. 7 th ed. National Academic Science, Washington, DC.

12. Sheldon IM, Lewis GS, LeBlanc S, Gilbert RO. Defining postpartum uterine disease in cattle. Theriogenology 2006;65:1516-1530.

13. SAS.SAS User`s Guide: Statistic (Version 9.3). Cary NC, USA Inst. Inc., 2010.

14. Hosmer DW, Lemeshow S. Applied logistic regression. New York, USA: Wiley; 1989.

15. López-Gatius F, García-Ispierto PI, Santolaria P, Yániz J, Nogareda C, López-Bejar M. Screening for high fertility in high-producing dairy cows. Theriogenology 2006;65:1678-1689.

16. Beagley JC, Whitman KJ, Baptiste KE, Scherzer J. Physiology and treatment of retained fetal membranes in cattle. J Vet Intern Med 2010;24:261-268. 
17. Slama H, Vaillancourt D, Goff AK. Metabolism of arachidonic acid by caruncular and allantochorionic tissues in cows with retained fetal membranes (RFM). Prostaglandins $1993 ; 45: 57-75$.

18. Wischral A, Verreschi ITN, Lima SB, Hayashi LF, Barnabe RC. Pre-parturition profile of steroids and prostaglandin in cows with or without foetal membrane retention. Anim Reprod Sci 2001;67:181-188.

19. Takagi M, Fujimoto S, Ohtani M, Miyamoto A, Wijagunawardane MPB, Acosta TJ, et al. Bovine retained placenta: Hormonal concentrations in fetal and maternal placenta. Placenta 2002;23:429-437.

20. Kimura K, Goff JP, Kehrli ME, Reinhardt TA. Decreased neutrophil function as a cause of retained placenta in dairy cattle. J Dairy Sci 2002;85:544-550.

21. Moretti P, Probo M, Morandi N, Trevisi E, Ferrari A, Minuti A, et al. Early post-partum hematological changes in Holstein dairy cows with retained placenta. Anim Reprod Sci 2015; 152:17-25.

22. Salgado EG, Bouda J, Cecilio AA, Doubek J, Velásquez FHF. Efecto de la aplicación de prostaglandina F2 $\alpha$ en las primeras horas posparto sobre las concentraciones séricas de calcio en vacas lecheras. Vet Méx 2014;1:1-13.

23. Reinhardt TA, Lippolis JD, McCluskey BJ, Goff JP, Horst RL. Prevalence of subclinical hypocalcaemia in dairy herds. Vet J 2011;188:122-124.

24. Whiteford LC, Sheldon IM. Association between clinical hypocalcaemia and postpartum endometritis. Vet Rec 2005;157:202-204.

25. Martinez N, Sinedino LDP, Bisinotto RS, Ribeiro ES, Gomes GC, Lima FS, et al. Effect of induced subclinical hypocalcaemia on physiological responses and neutrophil function in dairy cows. J Dairy Sci 2014;97:874-887.

26. Melendez P, Donovan GA, Risco CA, Goff JP. Plasma mineral and energy metabolite concentrations in dairy cows fed an anionic prepartum diet that did or did not have retained fetal membranes after parturition. Am J Vet Res 2004;65:1071-1076.

27. Han YK, Kim IH. Risk factors for retained placenta and the effect of retained placenta on the occurrence of postpartum diseases and subsequent reproductive performance in dairy cows. J Vet Sci 2005;6:53-59.

28. Sheldon IM, Price SB, Cronin J, Gilbert RO, Gadsby JE. Mechanisms of infertility associated with clinical and subclinical endometritis in high producing dairy cattle. Reprod Dom Anim 2009;44:1-9. 
29. Lima FS, Bisinotto RS, Ribeiro ES, Greco LF, Ayres H, Favoreto MG, et al. Effects of 1 or 2 treatments with prostaglandin $F_{2} \alpha$ on subclinical endometritis and fertility in lactating dairy cows inseminated by timed artificial insemination. J Dairy Sci 2013;96:6480-6488.

30. Chebel RC, Santos JEP, Reynolds JP, Cerri RLA, Juchem SO, Overton M. Factors affecting conception rate after artificial insemination and pregnancy loss in lactating dairy cows. Anim Reprod Sci 2004;84:239-255.

31. Orozco M, Gutiérrez CG, López R, Aguilar C, Roque C, Hernández-Cerón J. Pregnancy rate in dairy cows treated with progesterone for six days during estrus synchronization with PGF2 $\alpha$. Anim Reprod Sci 2016;166:128-132. 